\title{
Neurodegenerative drug discovery: building on the past, looking to the future
}

First draft submitted: 20 March 2017; Accepted for publication: 27 March 2017; Published online: 15 May 2017

Keywords: acetylcholinesterase inhibitors $\bullet$ drug discovery $\bullet$ monoamine oxidase inhibitors $\bullet$ multitarget directed ligands $\bullet$ natural antioxidants $\bullet$ neurodegenerative diseases - phosphodiesterase inhibitors • polypharmacology • proteinopathies $\bullet$ serotonin receptors

- small molecules • target-based approach

There are no therapeutic areas that are more pressing and frustrating than the neurodegenerative diseases. This is not just a common feeling of those like us tirelessly working in the field, but what clearly emerges from an analysis of more than two decades of intense academic and industrial research. Concretely speaking, in Alzheimer's disease (AD), no new treatments have been approved since 2003, and no new drugs that could bring relief to such an incredibly devastating disease are on the horizon. During the 2002-2012 period, 244 compounds were assessed in 413 trials for AD. Of those that progressed to Phase III, only memantine was approved for marketing (1.8\%), with an overall success rate for approval of $0.4 \%$ (99.6\% attrition) [1]. Verubecestat's flop in February 2017 was the latest blow in the hunt for a treatment [2]. Sadly, Parkinson's disease (PD), amyotrophic lateral sclerosis and related diseases share similar low success rates and gloomy outlooks. This means that all neurodegenerative diseases are presently incurable and only palliative treatments are available. What is even more daunting is that research is underfunded when compared with the huge economic and social costs of these devastating conditions. Indeed, despite the huge rewards of a successful treatment, in the last 5 years, large pharmaceutical companies have cut their programs by half [3].
Thus, it is our duty to give our best to pushing ahead and use all the knowledge acquired so far to solve this needle-in-haystack problem. As medicinal chemists, we are used to taking decisions while never quite having complete information. However, in neurodegeneration, answers to the most basic questions (What is the most promising target? What is the best approach? Single-target or multitarget?) have undoubtedly a higher degree of uncertainty than in other fields.

So, it seems perfectly logical that we should leave no stone unturned in the fight against neurodegenerative diseases and we should pursue any drug discovery approach that looks promising in terms of supportive hypothesis and clear objectives. In this context, this special issue of Future Medicinal Chemistry, which spans various approaches directed to various neurodegenerative diseases, is very timely and germane to current challenges. Specifically looking at the contributions, they can be grouped under two major categories. The recent reformulation of the cholinergic hypothesis [4] has renewed the interest in 'classical' AChE inhibitors. On the other side, scientists are moving towards more innovative approaches, aiming to discover new targets or new chemical entities acting simultaneously on more than one target. This latter approach is among the most followed ones in the neurodegeneration therapeutic area, building on the
Maria Laura Bolognesi

Department of Pharmacy \& Biotechnology, Alma Mater Studiorum, University of Bologna, Via Belmeloro 6, 40126 Bologna, Italy

marialaura.bolognesi@unibo.it
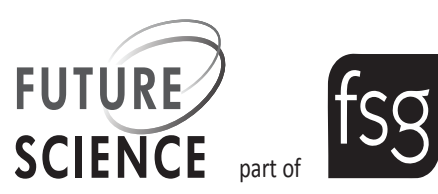
foundation that innovative multitarget-directed ligands (MTDLs) can more adequately address the complexity of these pathological conditions and overcome the challenges of a polypharmacological approach [5] .

Either in the case of a 'classical' or an innovative approach, we should challenge ourselves, ask questions incessantly and look at the problem from all the possible angles.

With this critical perspective, in their editorial, Gunesch and colleagues examine major approaches in the search of antineurodegenerative compounds, in other words, the search of multitarget small molecules bearing natural product-based features [6]. Indeed, many MTDLs developed in recent years, contain natural antioxidant fragments able to counteract the oxidative stress underlying neurodegeneration. However, the authors judiciously highlights how the combination of natural antioxidants with other pharmacophoric moieties, often leads to small molecules with disadvantageous pharmacokinetics and pharmacodynamics properties. Their personal (yet significant and shareable) opinion is that specific assays tailored on the chemical structure of the compounds under investigation, together with an objective interpretation of positive results that are needed to finally prove the approach reliability.

The potential of natural products in neurodegenerative diseases is confirmed by the review by Ramos et al., who accurately describe the pharmacological profile of hybrids inspired by melatonin [7]. Considering its lipophilicity and permeability through the blood-brain barrier, melatonin appears as a suitable scaffold for the search of multitarget therapies for AD. Although the developed hybrids show very promising results, once more it is clear that we need a more profound investigation of their mechanisms and safety profile, before such MTDLs can approach clinical trials.

The work of Rampa et al. is focused on the development of a series of naturally inspired chalcone-based carbamates, able to target cholinesterase enzymes and to show antioxidant activity [8]. Although three out of the four currently approved drugs for AD are AChE inhibitors, therapies aimed to restore the cholinergic deficit could only temporarily improve symptoms and cannot stop AD progression. Conversely, the developed chalcone-based carbamates might provide molecules able to simultaneously modulate two key targets of AD pathogenesis (i.e., $\mathrm{AChE}$ and oxidative stress), as the leaving fragment after cholinesterase carbamoylation shows cellular antioxidant activity.

Following a similar rationale, Ismaili and their research group proposed two different series of MTDLs against AD: benzochromenopyrimidinetriones and imidazopyridinotacrines [9,10]. Tacrine was the first marketed AChE inhibitor, however, it was withdrawn because of its hepatotoxicity. Nevertheless, it remains an important starting point for MTDL drug discovery endeavors, mainly due to its synthetic accessibility [11]. In this vein, Ismaili and their group considered this scaffold as a starting point for the search of so-called multipotent nontoxic tacrines, devoid of the parent drug's hepatotoxicity.

In addition to AChE, well-explored enzymatic targets are isoenzymes MAO-A and MAO-B. Thanks to the promising results obtained in $\mathrm{AD}$ clinical trials, several groups have been involved in the development of new hybrids able to inhibit ChE and MAO simultaneously. By extensively reviewing the literature, Knez et al. discuss recent positive examples in the field that seem to support the translational potential of this class [12].

Another AD symptomatic approach is based on the serotoninergic system modulation. As elegantly discussed by Lalut et al. [13], during the last decade the number of preclinical and clinical studies targeting the serotonin receptors $5-\mathrm{HT}_{4}$ and $5-\mathrm{HT}_{6}$ to treat cognitive deficits, has constantly increased. As a further step, several 5-HT receptor ligands have been tested in clinical trials in combination with AChE inhibitors. In parallel, some interesting MTDLs have been developed, which show promising results in preclinical characterization.

Clearly, targeting 'classical' enzymes and receptors involved in the pathogenesis of neurodegeneration is not the only feasible approach. Brahamchari et al. start from the well-accepted proposition that all neurodegenerative disorders are proteinopathies, as they are characterized by a common mechanism of misfolded proteins aggregation and accumulation [14]. In the light of this, a promising approach relies on the development of small drug-like entities able to interfere with the involved protein-protein interactions. The authors report on different classes of protein aggregation inhibitors and thoroughly describe the role of different functional groups in the inhibition process [15]. Importantly, they highlight the critical need for a comprehensive understanding of the disordered protein structural features for the development of an effective drug.

Looking at the future, Garcia et al. explore an innovative target by looking to neurodegeneration from an alternative perspective [16]. In their article, they focus on a neuroprotective approach for PD centered on the PD10A enzyme [16]. By exploiting a combined medicinal chemistry approach, for the first time the neuroprotective effect of phosphodiesterase $10 \mathrm{~A}$ inhibitors in PD cell-culture models have been clearly demonstrated.

All in all, I hope this issue I had the honor to guest edit will bring new impetus to the drug discovery professional community, so that we can face the current challenges building on the past, but keeping our eyes on the future. 


\section{Financial \& competing interests disclosure}

The author has no relevant affiliations or financial involvement with any organization or entity with a financial interest in or financial conflict with the subject matter or materials discussed in the manuscript. This includes employment,

\section{References}

1 Cummings JL, Morstorf T, Zhong K. Alzheimer's disease drug-development pipeline: few candidates, frequent failures. Alzheimers Res. Ther. 6(4), 37 (2014).

2 Another Alzheimer's drug flops in pivotal clinical trial. www.sciencemag.org

3 Alzheimer's Society. Ensuring the future of dementia research.

www.alzheimers.org.uk

4 Craig LA, Hong NS, Mcdonald RJ. Revisiting the cholinergic hypothesis in the development of Alzheimer's disease. Neurosci. Biobehav. Rev. 35(6), 1397-1409 (2011).

5 Bolognesi ML. Polypharmacology in a single drug: multitarget drugs. Curr. Med. Chem. 20(13), 1639-1645 (2013).

6 Gunesch S, Schramm S, Decker M. Natural antioxidants in hybrids for the treatment of neurodegenerative diseases - a successful strategy? Future Med. Chem. (2017).

7 Ramos E, Egea J, de los Rios C, Marco-Contelles J, Romero A. Melatonin as a versatile molecule to design novel multitarget hybrids against neurodegeneration. Future Med. Chem. (2017).

8 Rampa A, Monanari S, Pruccoli L et al. Chalcon-based carbamates for Alzheimer's disease treatment. Future Med. Chem. (2017).

9 Dgachi Y, Martin H, Bonet A et al. Synthesis and biological assessment of racemic benzochromenopyrimidine-triones consultancies, honoraria, stock ownership or options, expert testimony, grants or patents received or pending, or royalties.

No writing assistance was utilized in the production of this manuscript.

as promising agents for Alzheimer's disease therapy. Future Med. Chem. (2017).

10 Boulebd H, Ismaili L, Martin H et al. New (benz) imidazolopyridino tracrines as non hepatotoxic, cholinesterase inhibitors for Alzheimer disease. Future Med. Chem. (2017).

11 Nepovimova E, Uliassi E, Korabecny J et al. Multitarget drug design strategy: quinone-tacrine hybrids designed to block amyloid-beta aggregation and to exert anticholinesterase and antioxidant effects. J. Med. Chem. 57(20), 8576-8589 (2014).

12 Knez D, Sova M, Kosak U, Gobec S. Multi-targetdirected ligands for Alzheimer's disease: dual inhibitors of monoamine oxidase and cholinesterase. Future Med. Chem. (2017).

13 Lalut J, Karlia D, Dallemagne P, Rochais C. Modulating 5-HT4 and 5-HT6 receptors in Alzheimer's disease treatment. Future Med. Chem. (2017).

14 Eisenberg D, Jucker M. The amyloid state of proteins in human diseases. Cell 148(6), 1188-1203 (2012).

15 Brahmachari S, Paul A, Seagl D, Gazit E. Amyloid formation inhibition by small organic molecules: mechanistic insights and design rules. Future Med. Chem. (2017).

16 Garcia AM, Salado IG, Perez D et al. New pharmacological tools based on imidazole scaffold proved the utility of Phosphodiesterase 10 (PDE10) inhibitors for Parkinson's disease treatment. Future Med. Chem. (2017). 